https://doi.org/10.15407/ujpe66.7.570

M. MOLLA GESSESSE ${ }^{1,2}$

${ }^{1}$ Department of Physics, Aksum University

(P. O. Box 1010, Axum, Ethiopia; e-mail: mokemolla@gmail.com)

2 Department of Physics, University of Gondar

(P. O. Box 196, Gondar, Ethiopia)

\title{
INTERACTION OF TWO-LEVEL ATOMS WITH A SINGLE-MODE QUANTIZED RADIATION FIELD
}

\begin{abstract}
We have studied the statistical and squeezing properties of the cavity light generated by a twolevel laser. This optical system contains $N$ two-level atoms available in a cavity coupled to a single-mode vacuum reservoir. They are pumped to the top level from the bottom level by means of the electron bombardment. Applying the steady-state solutions of the equations of evolution of the expectation values of the atomic operators and the quantum Langevin equation, we obtained the global and local photon statistics of the single-mode light beam. We have found that, for the two-level laser operating well above the threshold, the uncertainties in the plus and minus quadratures are equal and satisfy the minimum uncertainty relation. In view of this, we have identified the light generated by the laser operating well above threshold to be coherent. On the other hand, the light generated by the laser operating at threshold is found to be chaotic. From the obtained results, we have also observed that a large part of the local mean photon number, the local photon number variance, and the local quadrature variance are confined in a relatively narrow frequency interval.
\end{abstract}

Ke ywords: mean photon number, quadrature variance, vacuum reservoir noise.

\section{Introduction}

The interactions of electromagnetic radiation with matter are the pillar for this work. It is then imperative to go through the fundamentals of the lightmatter interactions. Although the semiclassical approximation, where the field is assumed to be strong and treated classically, while the atoms are considered quantum-mechanical objects, gives remarkably good results in some systems, it is inadequate to provide information about the quantum-statistical properties of radiation. To this end, we start with a fully quantum- mechanical description of the interaction of single-mode electromagnetic radiation with an atom having two-energy levels.

The simplest quantum-mechanical model dealing with the atom-field interaction is the Jaynes-Cummings model, which describes an isolated two-level atom coupled to a single-mode quantized electromagnetic field resonant with the atomic transition $[1,2]$. The Jaynes-Cummings model has been studied extensively because of its relatively realistic description of the actual dipole coupling of an atom to

(C) M. MOLLA GESSESSE, 2021 a quantized radiation field [3]. Certain novel features of the system dynamics, such as the collapse and revival of the atomic coherence, were predicted. Some of these interesting effects have been observed experimentally. The interaction of a single-mode quantized radiation field with $N$ two-level atoms has been studied by many authors [3-5]. It has been shown that the fully quantum-mechanical model produces an incomplete set of linear evolution equations, the form of which depends on the number of atoms, as well as on the cavity light.

Fesseha [4] analyzed the quantum properties of the light generated by a two-level laser in which the twolevel atoms available in a closed cavity are pumped to the upper level by means of the electron bombardment. He carry out his analysis by putting the noise operators associated with a vacuum reservoir in the normal order. The normal ordering of the noise operators leads to the normally ordered quadrature and photon-number variance. According to his findings, the light generated by a two-level laser operating well above the threshold is coherent and the light generated by the same laser operating at the threshold is chaotic. But the quantum theory of a dissipative sys-

ISSN 2071-0194. Ukr. J. Phys. 2021. Vol. 66, No. 7 
tem has been a fascinating subject for theoreticians since the early day of quantum mechanics. The main reason for this interest is, of course, that any theory of quantum dissipation must necessarily be more complex than a classical theory. A consistent quantum theory of a damped system becomes possible, only when the model involves the fluctuating forces exerted on the system by the dissipation mechanism itself.

There are two general approaches to the subject. One deals with the dynamical equations for the density operators for the system. Whereas the other deals with the equation of motion for the dynamical operators of the system. Although the former approach is by far more common in the literature, the latter approach, however, offers the advantage that the Heisenberg equation of motion for the operators of the system has the same form as those for a classical damped system subject to the fluctuating forces, the Langevin equations, and, therefore, provide an easily visualizable picture of the damping process. It is the approach we will subject to use here.

Recently, Mekonnen [6] has also studied a threelevel laser in which three-level atoms available in a closed cavity are pumped from the bottom to the top level by coherent light. He carried out his analysis by putting the vacuum noise operators in arbitrary order. He has found that the light generated by the three-level laser operating under certain conditions is in a squeezed state, with the maximum quadrature squeezing being less than $43 \%$ below the vacuumstate level. In addition, he has also shown that the effect of the vacuum reservoir noise is to increase the photon number variance and to decrease the quadrature squeezing. However, the vacuum reservoir noise has no any effect on the mean photon number.

More recently, Mekonnen [7] (to be published very soon) has also studied a three-level laser in which three-level atoms available in an open cavity are pumped from the bottom and the ground levels to the top level by means of the electron bombardment. He carried out his analysis by putting the vacuum noise operators in an arbitrary order. He has found that the light generated by the three-level laser operating under certain conditions is in a squeezed state. In addition, he has established that although light modes $a$ and $b$ are separately in a chaotic state, it turns out that the two-mode cavity light is in a squeezed state under a certain condition, with a maximum quadrature squeezing of $46 \%$ below the coherent state level.
In this paper, we consider electrically pumped $N$ two-level atoms interacting with a single-mode quantized electromagnetic field at the resonance.

\section{Equations of Evolution}

Light is both radiated and absorbed by atoms, and the interaction between the quantized electromagnetic field and an atom represents one of the most fundamental problems in quantum optics. However, real atoms are complicated systems, and even the simplest real atom, the hydrogen atom, has a non-trivial energy level structure. It is therefore often necessary to approximate the behavior of a real atom by that of a much simpler quantum system. For many purposes, only two atomic energy levels play a significant role in the interaction with the electromagnetic field, so that it has become customary in many theoretical treatments to represent the atom by a quantum system with only two energy eigenstates. This is the most basic of all quantum systems, and it generally simplifies the treatment substantially.

In a real atom, the selection rules limit the allowed transitions between states, so that, in some cases, a certain state may couple to only a single one. Moreover, the optical pumping and electrical pumping techniques have been developed, which allows such preferred states to be prepared in the laboratory, and they have been successfully used in experiments. The two-level atom approximation is therefore close to the truth and not merely a mathematical convenience in some experimental situations [8].

\subsection{Single-Mode Cavity Light Damped by Reservoir}

We now turn to the formulation of the damping of a single radiation mode in a cavity by the aggregate of other modes in the reservoir. We now concentrate on the time development of the operators in the Heisenberg representation that characterize the dynamical system. The total Hamiltonian that describes the system-reservoir is given by [9]

$\hat{H}=\hat{H}_{S}+\hat{H}_{R}+\hat{H}_{S R}$.

This Hamiltonian can also be equally rewritten as

$\hat{H}=\hbar \omega \hat{b}^{\dagger} \hat{b}+\sum_{k} \hbar \omega_{k} \hat{a}_{k}^{\dagger} \hat{a}_{k}+\hbar \sum_{k}\left[\lambda_{k} \hat{b} \hat{a}_{k}^{\dagger}+\lambda_{k}^{*} \hat{b}^{\dagger} \hat{a}_{k}\right]$ 
Consistently with the general formalism, it is assumed that the reservoir oscillators are in thermal equilibrium, and the system operators $\hat{b}$ and $\hat{b}^{\dagger}$ commute with all reservoir operators $\hat{a}_{k}, \hat{a}_{k}^{\dagger}$. However, $\hat{b}$ and $\hat{b}^{\dagger}$, as well as $\hat{a}_{k}$, and $\hat{a}_{k}^{\dagger}$, satisfy the bosonic commutation rules. The equations of motion of the operators must satisfy the Heisenberg relation

$\dot{\hat{b}}=-\frac{i}{\hbar}[\hat{b}, \hat{H}]$.

In view of (2), the above equation takes the form

$\dot{\hat{b}}=-i \omega \hat{b}-i \sum_{k} \lambda_{k}^{*} \hat{a}_{k}$

Similarly,

$\dot{\hat{a}}_{k}=-i \omega_{k} \hat{a}_{k}-i \lambda_{k} \hat{b}$.

To solve these equations for the time-development of the system operators, we begin by integrating Eq. (5):

$\hat{a}_{k}(t)=\hat{a}_{k}\left(t_{0}\right) e^{-i \omega_{k}\left(t-t_{0}\right)}-i \int_{t_{0}}^{t} d t^{\prime} \lambda_{k} e^{-i \omega_{k}\left(t-t^{\prime}\right)} \hat{b}\left(t^{\prime}\right)$.

One then may insert Eq. (6) into Eq. (4), with $t_{0}=0$, to obtain the integro-differential equation

$$
\begin{aligned}
& \dot{\hat{b}}+i \omega \hat{b}+\sum_{k}\left|\lambda_{k}\right|^{2} \int_{0}^{t} d t^{\prime} e^{-i \omega_{k}\left(t-t^{\prime}\right)} \hat{b}\left(t^{\prime}\right)= \\
& =-i \sum_{k} \lambda_{k}^{*} \hat{a}_{k}(0) e^{-i \omega_{k} t} .
\end{aligned}
$$

At optical frequencies, the modes in a macroscopic cavity are densely packed, i.e., the number of modes per unit energy interval is very large. It is possible then to treat the term containing the integral by converting the sum over $k$ into an integral in which the discrete frequencies $\omega_{k}$ are replaced by a continuous frequency variable $\nu[9]$ :

$$
\sum_{k}\left|\lambda_{k}\right|^{2} e^{-i \omega_{k}\left(t-t^{\prime}\right)} \rightarrow \int_{-\infty}^{\infty} d \nu \lambda^{2}(\nu) e^{-i \nu\left(t-t^{\prime}\right)} .
$$

The major contribution to the interaction between the radiation mode and reservoir modes will arises from those modes whose frequencies are close to that of the radiation mode. In this narrow frequency interval, $\lambda^{2}(\nu)$ remains essentially constant. Then

$\int_{-\infty}^{\infty} d \nu \lambda^{2}(\nu) e^{-i \nu\left(t-t^{\prime}\right)} \simeq \lambda^{2}(\nu) \int_{-\infty}^{\infty} d \nu e^{-i \nu\left(t-t^{\prime}\right)}$.

This equation can also be rewritten as the sharp peak at $t \simeq t^{\prime}$ that allows us to extend the upper limit to infinity. We may also set

$$
\begin{aligned}
& \sum_{k}\left|\lambda_{k}\right|^{2} \int_{0}^{t} d t^{\prime} e^{-i \omega_{k}\left(t-t^{\prime}\right)} \hat{b}\left(t^{\prime}\right) \simeq \\
& \simeq 2 \pi \lambda^{2}(\nu) \int_{0}^{t} d t^{\prime} \hat{b}\left(t^{\prime}\right) \delta\left(t-t^{\prime}\right) .
\end{aligned}
$$

Analogously, we set

$$
\int_{0}^{\infty} f\left(x^{\prime}\right) \delta\left(x-x^{\prime}\right) d x^{\prime}=\frac{1}{2} f(x) .
$$

Hence, expression (10) reduces to

$$
\begin{aligned}
& \sum_{k}\left|\lambda_{k}\right|^{2} \int_{0}^{t} d t^{\prime} e^{-i \omega_{k}\left(t-t^{\prime}\right)} \hat{b}\left(t^{\prime}\right)= \\
& =\pi \lambda^{2}(\nu) \hat{b}(t)=\frac{\kappa}{2} \hat{b},
\end{aligned}
$$

where the cavity damping constant $\kappa$ is equal to $2 \pi \lambda^{2}(\nu)$.

Equation (7) can be now simplified to

$\dot{\hat{b}}+i \omega \hat{b}+\frac{\kappa}{2} \hat{b}=-i \sum_{k} \lambda_{k}^{*} \hat{a}_{k}(0) e^{-i \omega_{k} t} \equiv \hat{F}(t)$

and the Hermitian conjugate yields the corresponding equation for the creation operator

$\dot{\hat{b}}^{\dagger}-i \omega \hat{b}^{\dagger}+\frac{\kappa}{2} \hat{b}^{\dagger}=i \sum_{k} \lambda_{k} \hat{a}_{k}^{\dagger}(0) e^{i \omega_{k} t} \equiv \hat{F}^{\dagger}(t)$.

Since the reservoir operators are assumed to fluctuate, $\hat{F}(t)$ and $\hat{F}^{\dagger}(t)$, which are solely dependent on the reservoir operators $\hat{a}_{k}(0)$ and $\hat{a}_{k}^{\dagger}(0)$, must also fluctuate. The structure of these equations suggests therefore that they are of the Langevin type analogously to the equation for Brownian motion, and that $\kappa$ is to 
be interpreted as a damping constant; $\hat{F}(t)$ and $\hat{F}^{\dagger}(t)$ are also called Langevin noise sources.

The oscillator modes have been assumed to be in thermal equilibrium. Since

$\left\langle n_{k}\left|\hat{a}_{k}\right| n_{k}\right\rangle=0, \quad\left\langle n_{k}\left|\hat{a}_{k}^{\dagger}\right| n_{k}\right\rangle=0$,

where $\left|n_{k}\right\rangle$ is an eigenstate of the oscillator number operator $N_{k}=\hat{a}_{k}^{\dagger} \hat{a}_{k}$, we get

$\langle\hat{F}(t)\rangle=0 \quad$ and $\quad\left\langle\hat{F}^{\dagger}(t)\right\rangle=0$.

On the other hand,

$\left\langle\hat{a}_{k}^{\dagger} \hat{a}_{l}\right\rangle=\left\langle N_{k}\right\rangle \delta_{k l} \quad$ and $\quad\left\langle\hat{a}_{k} \hat{a}_{l}^{\dagger}\right\rangle=\left\langle N_{k}+1\right\rangle \delta_{k l}$.

Furthermore,

$\left\langle\hat{F}^{\dagger}(t) \hat{F}\left(t^{\prime}\right)\right\rangle=\sum_{k}\left|\lambda_{k}\right|^{2} e^{i \omega_{k}\left(t-t^{\prime}\right)}\left\langle N_{k}\right\rangle \simeq$

$\simeq \lambda^{2}(\nu)\langle n\rangle \int_{-\infty}^{\infty} e^{i \nu\left(t-t^{\prime}\right)}=$

$=2 \pi \lambda^{2}(\nu)\langle n\rangle \delta\left(t-t^{\prime}\right)=\kappa\langle n\rangle \delta\left(t-t^{\prime}\right)$,

where $\langle n\rangle$ is the average number of (reservoir) photons at the temperature $T$ that are nearly resonant with the radiation mode. In the same manner, it is found that

$$
\begin{aligned}
& \left\langle\hat{F}(t) \hat{F}^{\dagger}\left(t^{\prime}\right)\right\rangle=\sum_{k}\left|\lambda_{k}\right|^{2} e^{i \omega_{k}\left(t-t^{\prime}\right)}\left\langle N_{k}+1\right\rangle \simeq \\
& \simeq \lambda^{2}(\nu)\langle n+1\rangle \int_{-\infty}^{\infty} e^{i \nu\left(t-t^{\prime}\right)}= \\
& =2 \pi \lambda^{2}(\nu)\langle n+1\rangle \delta\left(t-t^{\prime}\right)=\kappa\langle n+1\rangle \delta\left(t-t^{\prime}\right) .
\end{aligned}
$$

Following the same line of reasoning, we can arrive at

$\left\langle\hat{F}(t) \hat{F}\left(t^{\prime}\right)\right\rangle=\left\langle\hat{F}^{\dagger}(t) \hat{F}^{\dagger}\left(t^{\prime}\right)\right\rangle=0$.

Remark. When the single mode cavity light is damped with the vacuum reservoir, we must substitute zero in place of the mean photon number of the thermal reservoir.

\subsection{Interaction Hamiltonian}

The Hamiltonian describing the interaction of one of the two-level atoms with the single-mode cavity light in the rotating frame of reference and involing the

ISSN 2071-0194. Ukr. J. Phys. 2021. Vol. 66, No. 7 rotating wave and electric dipole approximations is given by [10]

$\hat{H}=i g\left(\hat{\sigma}_{a}^{k \dagger} \hat{b}-\hat{b}^{\dagger} \hat{\sigma}_{a}^{k}\right)$,

where

$\hat{\sigma}_{a}^{k}=\left|b_{k}\right\rangle\left\langle a_{k}\right|$,

is the atomic lowering operator, $\hat{b}$ is the photonic annihilation operator, and $g$ is the coupling constant between the atom and the light. We assume that the radiation inside the cavity is coupled with the vacuum reservoir outside the cavity via single-port mirror. In addition, we carry out our calculation by putting the noise operators associated with the vacuum reservoir in arbitrary order. Thus, the noise operator will have some effect on the dynamics of the cavity mode operators.

\subsection{Quantum Langevin Equations}

We recall that the laser cavity is coupled to a singlemode vacuum reservoir via a single-port mirror. We can therefore write the quantum Langevin equation for the operator $\hat{b}$ as

$\frac{d \hat{b}}{d t}=-\frac{\kappa}{2} \hat{b}-i[\hat{b}, \hat{H}]+\hat{F}(t)$

where $\kappa$ is the cavity damping constant. Then, with the aid of Eq. (21), we easily find

$\frac{d \hat{b}}{d t}=-\frac{\kappa}{2} \hat{b}-g \hat{\sigma}_{a}^{k}+\hat{F}(t)$

This is the quantum Langevin equation for the cavity mode operator $\hat{b}$. We use of the Heisenberg equation of motion given by

$\frac{d}{d t}\langle\hat{A}\rangle=-i\langle[\hat{A}, \hat{H}]\rangle$,

$\frac{d}{d t}\left\langle\hat{\sigma}_{a}^{k}\right\rangle=g\left\langle\left(\hat{\eta}_{b}^{k}-\hat{\eta}_{a}^{k}\right) \hat{b}\right\rangle$,

$\frac{d}{d t}\left\langle\hat{\eta}_{a}^{k}\right\rangle=g\left\langle\left(\hat{\sigma}_{a}^{\dagger k} \hat{b}+\hat{b}^{\dagger} \hat{\sigma}_{a}^{k}\right)\right\rangle$,

$\frac{d}{d t}\left\langle\hat{\eta}_{b}^{k}\right\rangle=-g\left\langle\left(\hat{\sigma}_{a}^{\dagger k} \hat{b}+\hat{b}^{\dagger} \hat{\sigma}_{a}^{k}\right)\right\rangle$

where

$\hat{\eta}_{a}^{k}=\left|a_{k}\right\rangle\left\langle a_{k}\right|$,

$\hat{\eta}_{b}^{k}=\left|b_{k}\right\rangle\left\langle b_{k}\right|$. 
We see that Eqs. (26), (27), and (28), are incomplete linear coupled differential equations whose timedependent solutions are not known explicitly. Thus, to circumvent such a challenge, we apply the adiabatic elimination scheme [11]. To this end, expression (24) in this elimination scheme can be rewritten as

$\hat{b}(t)=-\frac{2 g \hat{\sigma}_{a}^{k}}{k}(t)+\frac{2 \hat{F}}{k}(t)$.

By substituting (31) back into (26), (27), and (28), we get

$\frac{d}{d t}\left\langle\hat{\sigma}_{a}^{k}\right\rangle=-\frac{\gamma_{c}}{2}\left\langle\hat{\sigma}_{a}^{k}\right\rangle+\frac{2 g}{k}\left[\left\langle\hat{\eta}_{b}^{k} \hat{F}(t)\right\rangle-\left\langle\hat{\eta}_{a}^{k} \hat{F}(t)\right\rangle\right]$,

$\frac{d}{d t}\left\langle\hat{\eta}_{a}^{k}\right\rangle=-\gamma_{c}\left\langle\hat{\eta}_{a}^{k}\right\rangle+\frac{2 g}{k}\left[\left\langle\hat{\sigma}_{a}^{k \dagger} \hat{F}(t)\right\rangle+\left\langle\hat{\sigma}_{a}^{k} \hat{F}_{a}^{\dagger}(t)\right\rangle\right]$,

$\frac{d}{d t}\left\langle\hat{\eta}_{b}^{k}\right\rangle=\gamma_{c}\left\langle\hat{\eta}_{a}^{k}\right\rangle-\frac{2 g}{k}\left[\left\langle\hat{\sigma}_{a}^{k \dagger} \hat{F}(t)\right\rangle+\left\langle\hat{\sigma}_{a}^{k} \hat{F}_{a}^{\dagger}(t)\right\rangle\right]$.

Following the same procedure presented in [6], we obtain

$\frac{d}{d t}\left\langle\hat{\sigma}_{a}^{k}\right\rangle=-\frac{\gamma_{c}}{2}\left\langle\hat{\sigma}_{a}^{k}\right\rangle$,

$\frac{d}{d t}\left\langle\hat{\eta}_{a}^{k}\right\rangle=-\gamma_{c}\left\langle\hat{\eta}_{a}^{k}\right\rangle$

$\frac{d}{d t}\left\langle\hat{\eta}_{b}^{k}\right\rangle=\gamma_{c}\left\langle\hat{\eta}_{a}^{k}\right\rangle$

where

$\gamma_{c}=\frac{4 g^{2}}{k}$

We prefer to call the parameter defined by Eq. (38) the stimulated emission decay constant [12]. Based on the definition of this decay constant, we infer that the atom in the upper level and inside a closed cavity emits a photon due to its interaction with the cavity light. We certainly identify this process to be a stimulated photon emission.

Now, we are in a position to include the contribution of all the $N$ atoms to the dynamics of the system of interest. We have

$\frac{d}{d t}\left\langle\hat{m}_{a}\right\rangle=-\frac{\gamma_{c}}{2}\left\langle\hat{m}_{a}\right\rangle$

$\frac{d}{d t}\left\langle\hat{N}_{a}\right\rangle=-\gamma_{c}\left\langle\hat{N}_{a}\right\rangle$,

$\frac{d}{d t}\left\langle\hat{N}_{b}\right\rangle=\gamma_{c}\left\langle\hat{N}_{a}\right\rangle$ where the operators $\hat{N}_{a}$ and $\hat{N}_{b}$ represent the number of atoms on the upper and lower levels. Furthermore, employing the completeness relation

$\hat{\eta}_{a}^{k}+\hat{\eta}_{b}^{k}=\hat{I}$

we easily arrive at

$\left\langle\hat{N}_{a}\right\rangle+\left\langle\hat{N}_{b}\right\rangle=N$.

In addition, using the definition given by Eq. (22), we get, for any $k$,

$\left\langle\hat{m}_{a}^{\dagger} \hat{m}_{a}\right\rangle=N\left\langle\hat{N}_{a}\right\rangle$

and

$\left\langle\hat{m}_{a} \hat{m}_{a}^{\dagger}\right\rangle=N\left\langle\hat{N}_{b}\right\rangle$.

When the cavity light interacts with all $N$ two-level atoms available in the cavity, the quantum Langevin equation is expressible as [13]

$\frac{d \hat{b}}{d t}=-\frac{k}{2} \hat{b}+\frac{g}{\sqrt{N}} \hat{m}_{a}+\hat{F}(t)$.

The two-level atoms available in the cavity are pumped from the lower level to the upper level by means of the electron bombardment. The pumping process must certainly affect the equation of evolution of the atomic operators [7]. Hence, we will consider the effect of the pumping process on the equation of evolution of the operators $\left\langle\hat{N}_{a}\right\rangle$ and $\left\langle\hat{N}_{b}\right\rangle$. To this end, we present Eqs. (40) and (41) in the form

$\frac{d}{d t}\left\langle\hat{N}_{a}\right\rangle=-\gamma_{c}\left\langle\hat{N}_{a}\right\rangle+r_{a}\left\langle\hat{N}_{b}\right\rangle$

$\frac{d}{d t}\left\langle\hat{N}_{b}\right\rangle=\gamma_{c}\left\langle\hat{N}_{a}\right\rangle-r_{a}\left\langle\hat{N}_{b}\right\rangle$

where $r_{a}$ is the rate at which a single atom is pumped to the upper level. Now, on the basis of expression (43), we can rewrite the above expressions as

$\frac{d}{d t}\left\langle\hat{N}_{a}\right\rangle=-\left(\gamma_{c}+r_{a}\right)\left\langle\hat{N}_{a}\right\rangle+r_{a} N$,

$\frac{d}{d t}\left\langle\hat{N}_{b}\right\rangle=-\left(\gamma_{c}+r_{a}\right)\left\langle\hat{N}_{b}\right\rangle+\gamma_{c} N$.

The steady-state solutions of these equations finally take the form

$\left\langle\hat{N}_{a}\right\rangle=\frac{r_{a} N}{\gamma_{c}+r_{a}}$

$\left\langle\hat{N}_{b}\right\rangle=\frac{\gamma_{c} N}{\gamma_{c}+r_{a}}$.

ISSN 2071-0194. Ukr. J. Phys. 2021. Vol. 66, No. 7 


\section{Photon Statistics}

The statistical properties of a light beam is described in terms of the mean photon number and the variance of the photon number. Here, we wish to calculate the mean photon number and the variance of photon number of the light generated by a two-level laser employing the steady-state solutions of the equations of evolution of the expectation values of the atomic operators and the quantum Langevin equation for the cavity mode operator.

\subsection{Global Mean Photon Number}

The photon number of the cavity light at any time is given by $\hat{b}^{\dagger} \hat{b}$ and the mean of the photon number is represented by $\left\langle\hat{b}^{\dagger} \hat{b}\right\rangle$. Here, we need to determine the global mean photon number of the cavity light. The equation of evolution of the mean photon number of the cavity light is as follows:

$\frac{d}{d t}\left\langle\hat{b}^{\dagger} \hat{b}\right\rangle=\left\langle\frac{d \hat{b}^{\dagger}}{d t} \hat{b}\right\rangle+\left\langle\hat{b}^{\dagger} \frac{d \hat{b}}{d t}\right\rangle$.

Making use of Eq. (46) and its adjoint, we have

$\frac{d}{d t}\left\langle\hat{b}^{\dagger} \hat{b}\right\rangle=-k\left\langle\hat{b}^{\dagger} \hat{b}\right\rangle+\frac{g}{\sqrt{N}}\left[\left\langle\hat{m}_{a}^{\dagger} \hat{b}\right\rangle+\left\langle\hat{b}^{\dagger} \hat{m}_{a}\right\rangle\right]+$

$+\left\langle\hat{F}^{\dagger}(t) \hat{b}(t)\right\rangle+\left\langle\hat{b}^{\dagger}(t) \hat{F}(t)\right\rangle$.

Now, we are interested to evaluate the above expressions one by one. Using the fact that the vacuum reservoir noise operator associated with the cavity mode operator at a certain time does not affect the atomic or the cavity mode operator at earlier times along with $\left\langle\hat{F}^{\dagger}(t) \hat{F}\left(t^{\prime}\right)\right\rangle=0$, one can arrive at

$\left\langle\hat{F}^{\dagger}(t) \hat{b}(t)\right\rangle=0$

and

$\left\langle\hat{m}_{a}^{\dagger}(t) \hat{b}(t)\right\rangle=\frac{2}{\kappa} \frac{g}{\sqrt{N}}\left\langle\hat{m}_{a}^{\dagger}(t) \hat{m}_{a}(t)\right\rangle$.

Substituting (55), (56), and their complex conjugates in expression (54), we get

$\frac{d}{d t}\left\langle\hat{b}^{\dagger} \hat{b}\right\rangle=-\kappa\left\langle\hat{b}^{\dagger} \hat{b}\right\rangle+\frac{4}{\kappa} \frac{g^{2}}{N}\left\langle\hat{m}_{a}^{\dagger} \hat{m}_{a}\right\rangle$.

With regard for Eqs. (38) and (44), the above equation finally takes the following form for a steady-state:

$\left\langle\hat{b}^{\dagger} \hat{b}\right\rangle=\frac{\gamma_{c}}{\kappa}\left\langle\hat{N}_{a}\right\rangle$.

ISSN 2071-0194. Ukr. J. Phys. 2021. Vol. 66, No. 7

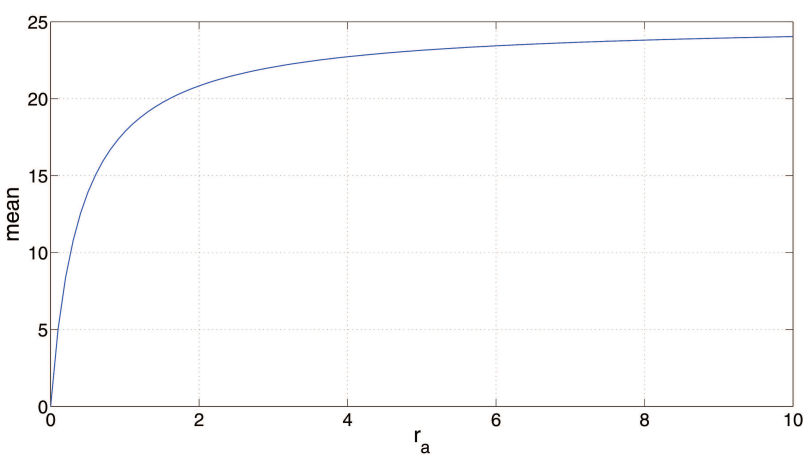

Fig. 1. Mean photon number [Eq. (59)] versus $r_{a}$ for $\gamma_{c}=0.4$, $\kappa=0.8$, and $N=50$

Now, with the aid of (51), the mean photon number of light mode $b$ is found to be

$\left\langle\hat{b}^{\dagger} \hat{b}\right\rangle=\frac{\gamma_{c}}{k}\left(\frac{r_{a} N}{\gamma_{c}+r_{a}}\right)$.

For the single-mode radiation operating well above threshold $\gamma_{c} \ll r_{a}$, Eq. (59) is reduced to

$\left\langle\hat{b}^{\dagger} \hat{b}\right\rangle=\frac{\gamma_{c}}{k} N$

For the same radiation field operating at the threshold $\gamma_{c}=r_{a}$, Eq. (59) can be rewritten as

$\left\langle\hat{b}^{\dagger} \hat{b}\right\rangle=\frac{\gamma_{c}}{2 k} N$.

\subsection{Local Mean Photon Number}

In this subsection, we are going to study the local mean photon number of the cavity light represented by light mode $b$. In order to determine the mean photon number of the cavity light in the given frequency interval, we must first consider the power spectrum of the cavity light. The power spectrum of the cavity light with central frequency $\omega_{0}$ is expressible as [14]

$P(\omega)=\frac{1}{\pi} \operatorname{Re} \int_{0}^{\infty} d \tau e^{i\left(\omega-\omega_{0}\right) \tau}\left\langle\hat{b}^{\dagger}(t) \hat{b}(t+\tau)\right\rangle_{s s}$.

Integrating both sides of Eq. (62) over $\omega$, we readily get

$\int_{-\infty}^{\infty} P(\omega) d \omega=\left\langle\hat{b}^{\dagger}(t) \hat{b}(t)\right\rangle_{s s}=\bar{n}$ 


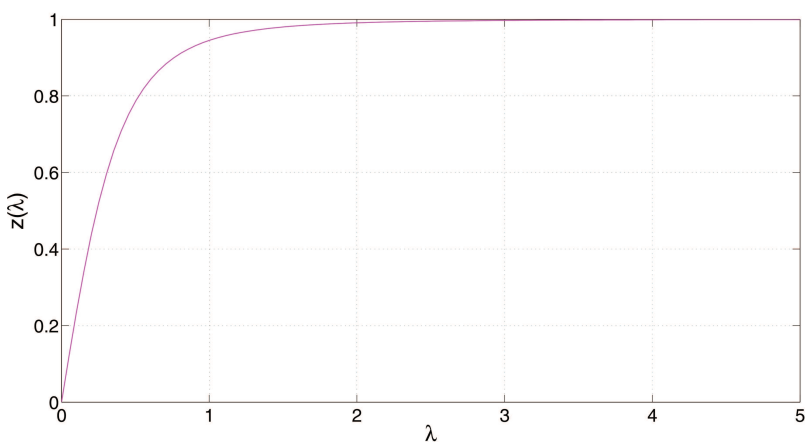

Fig. 2. Plot of the characteristic function $z(\lambda)$ [Eq. (70)] versus $\lambda$ for $\gamma_{c}=0.4$, and $\kappa=0.8$

in which $\bar{n}$ is the steady-state mean photon number of light mode $b$ [12]. From this result, we observe that $P(\omega) d \omega$ is the steady-state mean photon number of light mode $b$ in the frequency interval between $\omega$ and $\omega+d \omega[12]$.

The two-time correlation function that appears in expression (62) is found at a steady-state to be

$\left\langle\hat{b}^{\dagger}(t) \hat{b}(t+\tau)\right\rangle_{s s}=\bar{n}\left[\frac{\kappa}{\kappa-\gamma_{c}} e^{-\frac{\gamma_{c}}{2} \tau}-\frac{\gamma_{c}}{\kappa-\gamma_{c}} e^{-\frac{\kappa}{2} \tau}\right]$

Now on substituting Eq. (64) into (62), the power spectrum of light mode $b$ takes the form

$$
\begin{aligned}
& P(\omega)=\frac{\kappa \bar{n}}{\kappa-\gamma_{c}}\left[\frac{\gamma_{c} / 2 \pi}{\left(\omega-\omega_{0}\right)^{2}+\frac{\gamma_{c}^{2}}{2}}\right]- \\
& -\frac{\gamma_{c} \bar{n}}{\kappa-\gamma_{c}}\left[\frac{\kappa / 2 \pi}{\left(\omega-\omega_{0}\right)^{2}+\left(\frac{\kappa}{2}\right)^{2}}\right] .
\end{aligned}
$$

We recall that the mean photon number of light mode $b$ in the interval between $\omega^{\prime}=-\lambda$ and $\omega^{\prime}=\lambda$

Values of the $z(\lambda)$ and the corresponding

local mean photon number

\begin{tabular}{|l|l|l|}
\hline$\lambda$ & $z(\lambda)$ & $\bar{n}_{a \pm \lambda}$ \\
\hline 0.5 & 0.788 & $0.788 \bar{n}$ \\
1.0 & 0.946 & $0.946 \bar{n}$ \\
2.0 & 0.991 & $0.991 \bar{n}$ \\
3.0 & 0.997 & $0.997 \bar{n}$ \\
4.0 & 0.999 & $0.999 \bar{n}$ \\
\hline
\end{tabular}

is expressible as [12]

$\bar{n}_{ \pm \lambda}=\int_{-\lambda}^{\lambda} P\left(\omega^{\prime}\right) d \omega^{\prime}$

where $\omega^{\prime}=\omega-\omega_{0}$. Therefore, substituting (65) into (66), carrying out the integration, and making use of the relation

$\int_{-\lambda}^{\lambda} \frac{d x}{x^{2}+a^{2}}=\frac{2}{a} \tan ^{-1}\left(\frac{\lambda}{a}\right)$

we arrive at

$\bar{n}_{ \pm \lambda}=\bar{n}\left[\frac{2 \kappa / \pi}{\kappa-\gamma_{c}} \tan ^{-1}\left(\frac{\lambda}{\gamma_{c}}\right)-\frac{2 \gamma_{c} / \pi}{\kappa-\gamma_{c}} \tan ^{-1}\left(\frac{\lambda}{\kappa}\right)\right]$.

Now, the mean photon number of light mode $b$ in the frequency interval $\pm \lambda$ can be written as

$\bar{n}_{ \pm \lambda}=\bar{n} z(\lambda)$

where the function $z(\lambda)$ is given by

$z(\lambda)=\frac{2}{\pi}\left[\frac{\kappa}{\kappa-\gamma_{c}} \tan ^{-1}\left(\frac{\lambda}{\gamma_{c}}\right)-\right.$

$\left.-\frac{\gamma_{c}}{\kappa-\gamma_{c}} \tan ^{-1}\left(\frac{\lambda}{\kappa}\right)\right]$.

We see from Eq. (69) along with the plot of $z(\lambda)$ that $\bar{n}_{ \pm \lambda}$ increases with $\lambda$ until it reaches the maximum value given by Eq. (59). From the plot of Fig. 2, some of the values of $z(\lambda)$ and the corresponding local mean photon number $\bar{n}_{ \pm \lambda}$ are shown below.

\subsection{Global Photon-Number Variance}

Now, we will study the photon-number variance of light mode $b$ in the entire frequency interval. The photon-number variance of light mode $b$ at any suppressed time $t$ is given by

$\Delta n^{2}=\left\langle\left(\hat{b}^{\dagger} \hat{b}\right)^{2}\right\rangle-\left\langle\hat{b}^{\dagger} \hat{b}\right\rangle^{2}$

Applying the fact that $\hat{b}$ is a Gaussian variable, we get [14]

$\Delta n^{2}=\left\langle\hat{b}^{\dagger} \hat{b}\right\rangle\left\langle\hat{b} \hat{b}^{\dagger}\right\rangle+\left\langle\hat{b}^{\dagger 2}\right\rangle\left\langle\hat{b}^{2}\right\rangle$.

The equation of evolution for the operator $\left\langle\hat{b}^{2}\right\rangle$ can be expressed as

$\frac{d}{d t}\left\langle\hat{b}^{2}(t)\right\rangle=-\kappa\left\langle\hat{b}^{2}(t)\right\rangle$.

ISSN 2071-0194. Ukr. J. Phys. 2021. Vol. 66, No. 7 
Finally, the steady-state solution of the above equation is

$$
\left\langle\hat{b}^{2}\right\rangle=0 \text {. }
$$

Next, the equation of evolution for $\left\langle\hat{b}(t) \hat{b}^{\dagger}(t)\right\rangle$ takes the form

$\frac{d}{d t}\left\langle\hat{b} \hat{b}^{\dagger}\right\rangle=-\kappa\left\langle\hat{b} \hat{b}^{\dagger}\right\rangle+\kappa+\frac{\gamma_{c}}{N}\left\langle\hat{m}_{a} \hat{m}_{a}^{\dagger}\right\rangle$.

With the help of the relation indicated in (45), we find the steady-state solution of this equation to be

$$
\left\langle\hat{b} \hat{b}^{\dagger}\right\rangle_{s s}=\frac{\gamma_{c}}{\kappa}\left\langle\hat{N}_{b}\right\rangle+1 \text {. }
$$

Finally, in view of Eq. (58) along with (74) and (76), the photon-number variance of light mode $b$ in the steady state turns out to be

$\Delta n^{2}=\frac{\gamma_{c}}{\kappa}\left\langle\hat{N}_{a}\right\rangle\left[\frac{\gamma_{c}}{\kappa}\left\langle\hat{N}_{b}\right\rangle+1\right]$.

At the threshold $r_{a}=\gamma_{c}$, this can be rewritten as

$\Delta n^{2}=\bar{n}^{2}+\bar{n}$.

From (61) and (78), we conclude that the photon statistics of light mode $b$ is supper-Poissonian. We also note that light mode $b$ is in a chaotic state. It can also be verified that, well above the threshold, light mode $b$ is in a coherent state. In view of (51) and (52), the photon-number variance of light mode $b$ has, in the steady state, the form

$\Delta n^{2}=\frac{\gamma_{c}}{\kappa}\left[\frac{r_{a} N}{r_{a}+\gamma_{c}}\right]+\left[\frac{\gamma_{c}}{\kappa}\right]^{2}\left[\frac{r_{a} \gamma_{c} N^{2}}{\left(r_{a}+\gamma_{c}\right)^{2}}\right]$.

The plot in Fig. 3 indicates that fluctuations are increasing up to $r_{a}=0.4$ with the maximum value about 169 and then decreasing, accordingly.

\subsection{Local Photon-Number Variance}

In this subsection, we wish to calculate the photonnumber variance for light mode $b$ in a given frequency interval. To determine the photon-number variance for light mode $b$, we consider the spectrum of photonnumber fluctuations for light mode $b$. The spectrum of photon-number fluctuations for light mode $b$ with the central frequency $\omega_{0}$ is expressible as [4]

$S(\omega)=\frac{1}{\pi} \int_{0}^{\infty} d \tau e^{i\left(\omega-\omega_{0}\right) \tau}\langle\hat{n}(t), \hat{n}(t+\tau)\rangle_{s s}$,

ISSN 2071-0194. Ukr. J. Phys. 2021. Vol. 66, No. 7

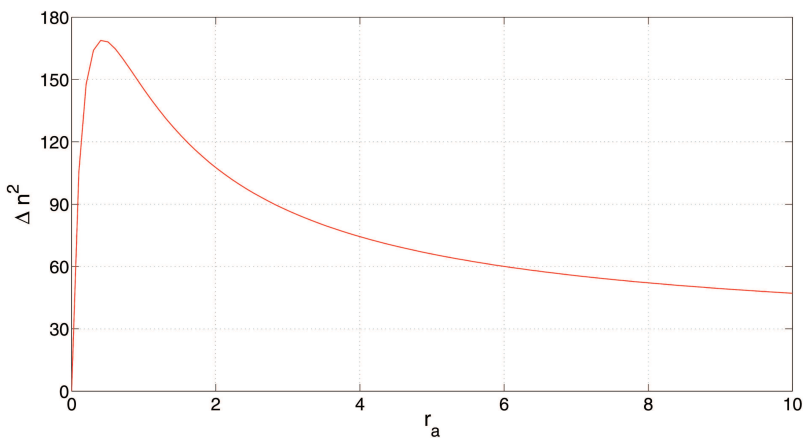

Fig. 3. Plot of the photon number variance [Eq. (79)] versus $r_{a}$ for $\gamma_{c}=0.4, \kappa=0.8$, and $N=50$

where

$\hat{n}(t)=\hat{b}^{\dagger}(t) \hat{b}(t)$

and

$\hat{n}(t+\tau)=\hat{b}^{\dagger}(t+\tau) \hat{b}(t+\tau)$.

Integrating both sides of Eq. (80) over $\omega$, we easily find

$\int_{-\infty}^{\infty} S(\omega) d \omega=\Delta n^{2}$

where $\Delta n^{2}$ is the photon-number variance of light mode $b$ in the steady state. We can then assert that $S(\omega) d \omega$ is the photon-number variance for light mode $b$ in the interval between $\omega$ and $\omega+d \omega$ in the steady state [12].

With regard for Eqs. (81) and (82), two-time correlation function is found to be

$\langle\hat{n}(t), \hat{n}(t+\tau)\rangle=\left\langle\hat{b}^{\dagger}(t) \hat{b}(t+\tau)\right\rangle\left\langle\hat{b}(t) \hat{b}^{\dagger}(t+\tau)\right\rangle+$

$+\langle\hat{b}(t) \hat{b}(t+\tau)\rangle\left\langle\hat{b}^{\dagger}(t) \hat{b}^{\dagger}(t+\tau)\right\rangle$

where

$\left\langle\hat{b}(t) \hat{b}^{\dagger}(t+\tau)\right\rangle=\left\langle\hat{b}(t) \hat{b}^{\dagger}(t)\right\rangle \times$

$\times\left[\frac{\kappa}{\kappa-\gamma_{c}} e^{-\frac{\gamma_{c}}{2} \tau}-\frac{\gamma_{c}}{\kappa-\gamma_{c}} e^{-\frac{1}{2} \kappa \tau}\right]-$

$-\frac{\kappa}{\kappa-\gamma_{c}}\left[e^{-\frac{\gamma_{c}}{2} \tau}-e^{-\frac{\kappa}{2} \tau}\right]$,

$\langle\hat{b}(t) \hat{b}(t+\tau)\rangle=\langle\hat{b}(t) \hat{b}(t)\rangle \times$

$\times\left[\frac{\kappa}{\kappa-\gamma_{c}} e^{-\frac{\gamma_{c}}{2} \tau}-\frac{\gamma_{c}}{\kappa-\gamma_{c}} e^{-\frac{1}{2} \kappa \tau}\right]$, 


$$
\begin{aligned}
& \left\langle\hat{b}^{\dagger}(t) \hat{b}^{\dagger}(t+\tau)\right\rangle=\left\langle\hat{b}^{\dagger}(t) \hat{b}^{\dagger}(t)\right\rangle \times \\
& \times\left[\frac{\kappa}{\kappa-\gamma_{c}} e^{-\frac{\gamma_{c}}{2} \tau}-\frac{\gamma_{c}}{\kappa-\gamma_{c}} e^{-\frac{1}{2} \kappa \tau}\right] .
\end{aligned}
$$

Substituting Eqs. (64), (85), (86), and (87) into (84), we get, in the steady state,

$$
\begin{aligned}
& \langle\hat{n}(t), \hat{n}(t+\tau)\rangle_{s s}=\left[\left\langle\hat{b}^{\dagger 2}\right\rangle\left\langle\hat{b}^{2}\right\rangle+\left\langle\hat{b}^{\dagger} \hat{b}\right\rangle\left\langle\hat{b} \hat{b}^{\dagger}\right\rangle\right] \times \\
& \times\left[\left(\frac{\kappa}{\kappa-\gamma_{c}}\right)^{2} e^{-\gamma_{c} \tau}-\frac{2 \kappa \gamma_{c}}{\left(\kappa-\gamma_{c}\right)^{2}} e^{-\frac{1}{2}\left(\kappa+\gamma_{c}\right) \tau}+\right. \\
& \left.+\left(\frac{\gamma_{c}}{\kappa-\gamma_{c}}\right)^{2} e^{-\kappa \tau}\right]-\frac{\left\langle\hat{b}^{\dagger} \hat{b}\right\rangle}{\left(\kappa-\gamma_{c}\right)^{2}} \times \\
& \times\left[\kappa^{2} e^{-\gamma_{c} \tau}-\left(\kappa^{2}+\kappa \gamma_{c}\right) e^{-1 / 2\left(\kappa+\gamma_{c}\right) \tau}+\kappa \gamma_{c} e^{-\kappa \tau}\right] .
\end{aligned}
$$

Therefore, in view of this expression, the spectrum of photon-number fluctuations takes the form

$$
\begin{aligned}
& S(\omega)=\left(\frac{\Delta n}{\kappa-\gamma_{c}}\right)^{2}\left[\frac{\kappa^{2}}{\pi} \operatorname{Re} \int_{0}^{\infty} d \tau e^{-\left[\gamma_{c}-i\left(\omega-\omega_{0}\right)\right] \tau}-\right. \\
& -\frac{2 \gamma_{c} \kappa}{\pi} \operatorname{Re} \int_{0}^{\infty} d \tau e^{-\left[\frac{1}{2}\left(\kappa+\gamma_{c}\right)-i\left(\omega-\omega_{0}\right)\right] \tau}+ \\
& \left.+\frac{\gamma_{c}^{2}}{\pi} \operatorname{Re} \int_{0}^{\infty} d \tau e^{-\left[\kappa-i\left(\omega-\omega_{0}\right)\right] \tau}\right]- \\
& -\frac{\bar{n}}{\left(\kappa-\gamma_{c}\right)^{2}}\left[\frac{\kappa^{2}}{\pi} \operatorname{Re} \int_{0}^{\infty} d \tau e^{-\left[\gamma_{c}-i\left(\omega-\omega_{0}\right)\right] \tau}-\right. \\
& -\left(\frac{\kappa^{2}+\kappa \gamma_{c}}{\pi}\right) \operatorname{Re} \int_{0}^{\infty} d \tau e^{-\left[\frac{1}{2}\left(\kappa+\gamma_{c}\right)-i\left(\omega-\omega_{0}\right)\right] \tau}+ \\
& \left.+\frac{\kappa \gamma_{c}}{\pi} \operatorname{Re} \int_{0}^{\infty} d \tau e^{-\left[\kappa-i\left(\omega-\omega_{0}\right)\right] \tau}\right] .
\end{aligned}
$$

Performing the integration, we get

$$
\begin{aligned}
& S(\omega)=\Delta n^{2}\left[\left\{\frac{\kappa}{\kappa-\gamma_{c}}\right\}^{2}\left\{\frac{\frac{\gamma_{c}}{\pi}}{\gamma_{c}^{2}+\left(\omega-\omega_{0}\right)^{2}}\right\}-\right. \\
& -\frac{2 \kappa \gamma_{c}}{\left(\kappa-\gamma_{c}\right)^{2}}\left\{\frac{\frac{\kappa+\gamma_{c}}{2 \pi}}{\left(\frac{\kappa+\gamma_{c}}{2}\right)^{2}+\left(\omega-\omega_{0}\right)^{2}}\right\}+ \\
& \left.+\left\{\frac{\gamma_{c}}{\kappa-\gamma_{c}}\right\}^{2}\left\{\frac{\frac{\kappa}{\pi}}{\kappa^{2}+\left(\omega-\omega_{0}\right)^{2}}\right\}\right]= \\
& =-\bar{n}\left[\left\{\frac{\kappa}{\kappa-\gamma_{c}}\right\}^{2}\left\{\frac{\frac{\gamma_{c}}{\pi}}{\gamma_{c}^{2}+\left(\omega-\omega_{0}\right)^{2}}\right\}+\right.
\end{aligned}
$$

$$
\begin{aligned}
& +\frac{\kappa \gamma_{c}}{\left(\kappa-\gamma_{c}\right)^{2}}\left\{\frac{\frac{\kappa}{\pi}}{\kappa^{2}+\left(\omega-\omega_{0}\right)^{2}}\right\}- \\
& \left.-\frac{\kappa\left(\kappa+\gamma_{c}\right)}{\left(\kappa-\gamma_{c}\right)^{2}}\left\{\frac{\frac{\kappa+\gamma_{c}}{2 \pi}}{\left(\frac{\kappa+\gamma_{c}}{2}\right)^{2}+\left(\omega-\omega_{0}\right)^{2}}\right\}\right] .
\end{aligned}
$$

We recall that the local photon-number variance of the single-mode cavity light in the interval between $\omega^{\prime}=-\lambda$ and $\omega^{\prime}=\lambda$ is expressible as [15]

$$
\Delta n_{ \pm \lambda}^{2}=\int_{-\lambda}^{\lambda} S\left(\omega^{\prime}\right) d \omega^{\prime}
$$

in which $\omega^{\prime}=\omega-\omega_{0}$. , Therefore, substituting Eq. (90) into (91) and carrying out the integration, we arrive at

$$
\begin{aligned}
& \Delta n_{ \pm \lambda}^{2}=\Delta n^{2}\left[\frac{2}{\pi}\left(\frac{\kappa}{\kappa-\gamma_{c}}\right)^{2} \tan ^{-1}\left(\frac{\lambda}{\gamma_{c}}\right)-\right. \\
& -\frac{2}{\pi} \frac{2 \kappa \gamma_{c}}{\left(\kappa-\gamma_{c}\right)^{2}} \tan ^{-1}\left(\frac{2 \lambda}{\kappa+\gamma_{c}}\right)+ \\
& \left.+\frac{2}{\pi}\left(\frac{\gamma_{c}}{\kappa-\gamma_{c}}\right)^{2} \tan ^{-1}\left(\frac{\lambda}{\kappa}\right)\right]- \\
& -\bar{n}\left[\frac{2}{\pi}\left(\frac{\kappa}{\kappa-\gamma_{c}}\right)^{2} \tan ^{-1}\left(\frac{\lambda}{\gamma_{c}}\right)+\right. \\
& +\frac{2}{\pi} \frac{\kappa \gamma_{c}}{\left(\kappa-\gamma_{c}\right)^{2}} \tan ^{-1}\left(\frac{\lambda}{\kappa}\right)- \\
& \left.-\frac{2}{\pi} \frac{\kappa\left(\kappa+\gamma_{c}\right)}{\left(\kappa-\gamma_{c}\right)^{2}} \tan ^{-1}\left(\frac{2 \lambda}{\kappa+\gamma_{c}}\right)\right] .
\end{aligned}
$$

From the plot in Fig. 4, we notice that the local photon-number variance approaches the global photon-number variance, as $\lambda$ increases.

\section{Quadrature Variance}

Here, we study the quadrature variance of the cavity light produced by electrically bombarded twolevel atoms in a closed cavity and coupled to a vacuum reservoir via a single-port mirror. Applying the steady-state solutions of the equations of evolution of the expectation values of the atomic operators and the quantum Langevin equation for the cavity mode operator, we obtain the global and local quadrature variances of the single-mode cavity light.

ISSN 2071-0194. Ukr. J. Phys. 2021. Vol. 66, No. 7 


\subsection{Global Quadrature Variance}

We now calculate the global quadrature variances of light mode $b$, produced by the system under consideration. The squeezing properties of light mode $b$ is described by two quadrature operators [6]

$\hat{b}_{+}=\hat{b}^{\dagger}+\hat{b}$

and

$\hat{b}_{-}=i\left(\hat{b}^{\dagger}-\hat{b}\right)$.

These operators are Hermitian and satisfy the commutation relation

$\left[\hat{b}_{-}, \hat{b}_{+}\right]=2 i\left[\frac{\gamma_{c}}{\kappa}\left(\hat{N}_{a}-\hat{N}_{b}\right)-1\right]$.

The operators $\hat{b}_{+}$and $\hat{b}_{-}$represent physical quantities called the plus and minus quadratures, respectively. The uncertainty relation for the quadrature operators can be expressed as [16]

$\Delta \hat{b}_{+} \Delta \hat{b}_{-} \geq\left|\frac{\gamma_{c}}{\kappa}\left(\left\langle\hat{N}_{a}\right\rangle-\left\langle\hat{N}_{b}\right\rangle\right)-1\right|$,

so that, in view of Eqs. (51) and (52), we find the uncertainty relation for the quadrature operators of cavity light mode $b$ in the steady-state:

$\Delta \hat{b}_{+} \Delta \hat{b}_{-} \geq\left|\frac{\gamma_{c}}{\kappa}\left(\frac{r_{a}-\gamma_{c}}{r_{a}+\gamma_{c}}\right) N-1\right|$.

Moreover, we consider the case where the rate at which the bombarded atoms transit from the bottom level to the top one is much less than the stimulated emission decay constant. Hence, for $r_{a} \ll \gamma_{c}$, Eq. (97) reduces to

$\Delta \hat{b}_{+} \Delta \hat{b}_{-} \geq \frac{\gamma_{c}}{\kappa} N+1$.

Next, we proceed to calculate the quadrature variance of light mode $b$. The variance of the quadrature operators is expressible as

$\Delta \hat{b}_{ \pm}^{2}=\left\langle\hat{b}_{ \pm}^{2}\right\rangle-\left\langle\hat{b}_{ \pm}\right\rangle^{2}$.

The explicit form of the quadrature variance of the plus and minus quadratures can be expressed as

$\Delta \hat{b}_{+}^{2}=\left\langle\hat{b}_{+}^{2}\right\rangle-\left\langle\hat{b}_{+}\right\rangle^{2}$

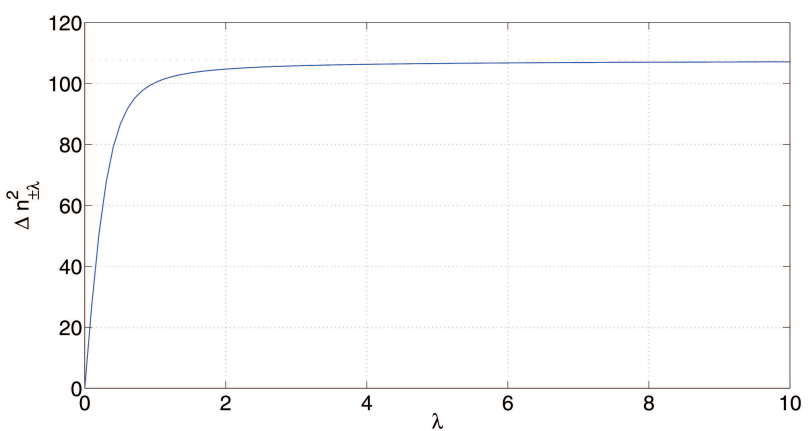

Fig. 4. Plot of $(\Delta n)_{ \pm \lambda}^{2}$ versus $\lambda$ for $\gamma_{c}=0.4, \kappa=0.8, r_{a}=2$, and $N=50$

and

$\left(\Delta \hat{b}_{-}\right)^{2}=\left\langle\hat{b}_{-}^{2}\right\rangle-\left\langle\hat{b}_{-}\right\rangle^{2}$.

With the aid of Eqs. (93) and (94), the above expressions take the form

$\Delta \hat{b}_{ \pm}^{2}=\left\langle\hat{b}^{\dagger} \hat{b}^{\dagger}+\left\langle\hat{b}^{\dagger} \hat{b}\right\rangle \pm\left\langle\hat{b}^{2}\right\rangle \pm\left\langle\hat{b}^{\dagger 2}\right\rangle \mp\right.$

$\mp\langle\hat{b}\rangle^{2} \mp\left\langle\hat{b}^{\dagger}\right\rangle^{2}-2\langle\hat{b}\rangle\left\langle\hat{b}^{\dagger}\right\rangle$.

Since $\hat{b}$ is a Gaussian variable with zero mean, we readily get

$\Delta \hat{b}_{ \pm}^{2}=\left\langle\hat{b} \hat{b}^{\dagger}\right\rangle+\left\langle\hat{b}^{\dagger} \hat{b}\right\rangle \pm\left\langle\hat{b}^{2}\right\rangle \pm\left\langle\hat{b}^{\dagger 2}\right\rangle$.

In view of Eqs. (58), (74), and (76), the quadrature variance of light mode $b$ takes, in the steady-state, the form

$\Delta \hat{b}_{ \pm}^{2}=\frac{\gamma_{c}}{\kappa}\left[\left\langle\hat{N}_{a}\right\rangle+\left\langle\hat{N}_{b}\right\rangle\right]+1$

Thus, the substitution of Eqs. (51) and (58) into Eq. (104) results in

$\Delta \hat{b}_{+}^{2}=\Delta \hat{b}_{-}^{2}=\frac{\gamma_{c}}{\kappa} N+1$.

At the threshold, the above expression is just equal to

$\Delta \hat{b}_{+}^{2}=\Delta \hat{b}_{-}^{2}=2 \bar{n}+1$.

This represents the quadrature variance of cavity light mode $b$. This indicates that light mode $b$ is in a chaotic state. In view of (98) and (105), we see that the uncertainties in the plus and minus quadratures are equal to each other and satisfy the minimum uncertainty relation. 


\subsection{Local Quadrature Variance}

We finally seek to obtain the quadrature variance of the cavity light in a given frequency interval. To this end, we firstly determine the spectrum of quadrature fluctuations of the single-mode cavity light. We define this spectrum with central frequency $\omega_{0}$ by [4]

$S_{ \pm}(\omega)=\frac{1}{\pi} \operatorname{Re} \int_{0}^{\infty}\left\langle\hat{b}_{ \pm}(t), \hat{b}_{ \pm}(t+\tau)\right\rangle_{s s} e^{i\left(\omega-\omega_{0}\right) \tau} d \tau$.

Integrating both sides of Eq. (107) over $\omega$, we get

$\int_{-\infty}^{\infty} S_{ \pm}(\omega) d \omega=\Delta b_{ \pm}^{2}$

where

$\Delta b_{ \pm}^{2}=\left\langle\hat{b}_{ \pm}(t), \hat{b}_{ \pm}(t)\right\rangle_{s s}$

is the quadrature variance of the light mode in the steady state. On the basis of the result given by (109), we assert that $S_{ \pm}(\omega) d \omega$ is the quadrature variance of the light mode in the steady state in the interval between $\omega$ and $\omega+d \omega$.

Now, the two-time correlation function involved in (107) is found to be

$$
\begin{aligned}
& \left\langle\hat{b}_{ \pm}(t), \hat{b}_{ \pm}(t+\tau)\right\rangle= \\
& =\Delta b_{ \pm}^{2}\left[\frac{\kappa}{\kappa-\gamma_{c}} e^{-\frac{\gamma_{c} \tau}{2}}-\frac{\gamma_{c}}{\kappa-\gamma_{c}} e^{-\frac{\kappa \tau}{2}}\right]- \\
& -\frac{\kappa}{\kappa-\gamma_{c}}\left[e^{-\frac{\gamma_{c}}{2} \tau}-e^{-\frac{\kappa}{2} \tau}\right] N .
\end{aligned}
$$

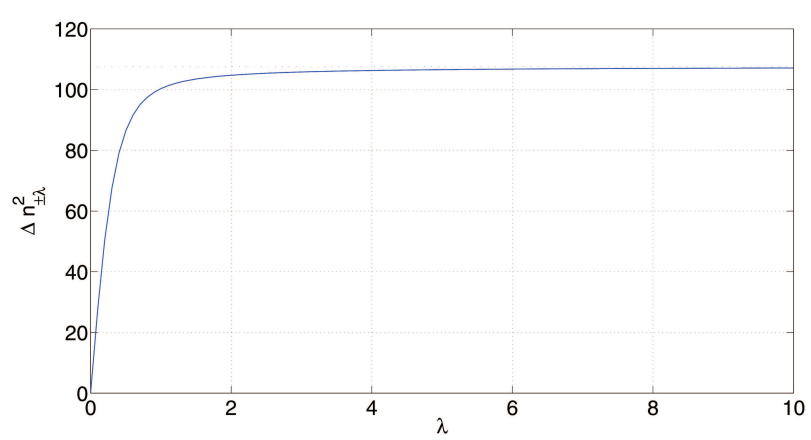

Fig. 5. Plot of the local quadrature variance of the two-mode cavity light versus $\lambda$ [Eq. (113)] for $\kappa=0.8, N=50$, and $\gamma_{c}=0.4$
Substituting this equation into (107) and carrying out the integration, the spectrum of quadrature fluctuations for the single-mode cavity light is as follows:

$$
\begin{aligned}
& S_{ \pm}(\omega)=\Delta b_{ \pm}^{2}\left[\frac{\kappa}{\kappa-\gamma_{c}}\left\{\frac{\frac{\gamma_{c}}{2 \pi}}{\left(\omega-\omega_{0}\right)^{2}+\left(\frac{\gamma_{c}}{2}\right)^{2}}\right\}-\right. \\
& \left.-\frac{\gamma_{c}}{\kappa-\gamma_{c}}\left\{\frac{\frac{\kappa}{2 \pi}}{\left(\omega-\omega_{0}\right)^{2}+\left(\frac{\kappa}{2}\right)^{2}}\right\}\right]- \\
& -\left[\frac{2 \kappa}{\kappa-\gamma_{c}}\left\{\frac{\frac{\gamma_{c}}{2 \pi}}{\left(\omega-\omega_{0}\right)^{2}+\left(\frac{\gamma_{c}}{2}\right)^{2}}\right\}-\right. \\
& \left.-\frac{\kappa}{\kappa-\gamma_{c}}\left\{\frac{\frac{\kappa}{2 \pi}}{\left(\omega-\omega_{0}\right)^{2}+\left(\frac{\kappa}{2}\right)^{2}}\right\}\right] .
\end{aligned}
$$

We realize that the quadrature variance in the frequency interval between $\omega^{\prime}=-\lambda$ and $\omega^{\prime}=\lambda$ is expressible as

$\Delta b_{ \pm \lambda}^{2}=\int_{-\lambda}^{\lambda} S_{ \pm}\left(\omega^{\prime}\right) d \omega^{\prime}$

in which $\omega^{\prime}=\omega-\omega_{0}$. Now, taking (111) into account and carrying out the integration, we easily obtain

$$
\begin{aligned}
& \Delta b_{ \pm \lambda}^{2}=\Delta b_{ \pm}^{2}\left[\frac{2 \kappa}{\pi\left(\kappa-\gamma_{c}\right)} \tan ^{-1}\left(\frac{2 \lambda}{\gamma_{c}}\right)-\right. \\
& \left.-\frac{2 \gamma_{c}}{\pi\left(\kappa-\gamma_{c}\right)} \tan ^{-1}\left(\frac{2 \lambda}{\kappa}\right)\right]- \\
& -\left[\frac{4 \kappa}{\pi\left(\kappa-\gamma_{c}\right)} \tan ^{-1}\left(\frac{2 \lambda}{\gamma_{c}}\right)-\frac{4 \kappa}{\pi\left(\kappa-\gamma_{c}\right)} \tan ^{-1}\left(\frac{2 \lambda}{\kappa}\right)\right] .
\end{aligned}
$$

From Fig. 5, we can clearly observe that the local quadrature variance approaches the global quadrature variance, as the frequency difference increases.

\section{Conclusion}

We have studied the statistical and squeezing properties of the cavity light generated by a two-level laser. In this optical system, $N$ two-level atoms available in a cavity coupled to a single-mode vacuum reservoir are pumped to the top level from the bottom one by means of the electron bombardment. Although we consider the case where the cavity light is interacting with the single-mode vacuum reservoir,

ISSN 2071-0194. Ukr. J. Phys. 2021. Vol. 66, No. 7 
we omit the case where the atoms inside the cavity are interacting with the single-mode vacuum reservoir.

Employing the interaction Hamiltonian, we have obtained the quantum Langevin equation for the cavity mode operator and the equation of evolution for the atomic operators. Based on the definition of the stimulated emission decay constant, we infer that an atom at the upper level and inside a closed cavity emits a photon due to its interaction with the cavity light. We certainly identify this process to be the stimulated emission.

Applying the steady-state solutions of the equations of evolution of the expectation values of the atomic operators and the quantum Langevin equation, we obtained the global and local photon statistics of the single-mode light beam. We have found that, for the two-level laser operating well above threshold, the uncertainties in the plus and minus quadratures are the same and satisfy the minimum uncertainty relation. In view of this, we have identified the light generated by the laser operating well above the threshold to be coherent. On the other hand, the light generated by the laser operating at the threshold is found to be chaotic. From the results we have obtained, we have also observed that a large part of the local mean photon number, as well as the local photon number variance along with the local quadrature variance, are confined in a relatively very small frequency interval.

1. M.S. Iqbal, S. Mahmood, M.S.K. Razmi, M.S. Zubairy. Interaction of two two-level atoms with a single-mode quantized radiation field. J. Opt. Soc. America B 5, 1312 (1988).

2. B.W. Shore, P.L. Knight. The Jaynes-Cummings model. J. Modern Optics 40, 1195 (1993).

3. E. Alebachew, K. Fesseha. Interaction of a two-level atom with squeezed light. Opt. Commun. 271, 154 (2007).

4. F. Kassahun. Stimulated emission by two-level atoms pumped to the upper level. Opt. Commun. 284, 1357 (2011).

5. T. Abebe, S. Mosissa, N. Belay. Coherently driven twolevel atom in open space and interacting with vacuum modes. Bulg. J. Phys. 46, 214 (2019).

6. M. Molla Gessesse. The noise effect of vacuum reservoir on the dynamics of three-level laser pumped by coherent light. Ukr. J. Phys. 65, 385 (2020).
7. M. Molla Gessesse. Electrically pumped two-mode laser dynamics. Ukr. J. Phys. 66, 206 (2021).

8. S. M. Barnett, P.M. Radmore. Methods in Theoretical Quantum Optics (Oxford University Press, 1997).

9. M. Weissbluth. Photon-Atom Interactions (United Kingdom Edition, 1989).

10. G.S. Agarwal. Quantum Optics (Cambridge University Press, 2013).

11. W. Theiss, U.M. Titulaer. The systematic adiabatic limination of fast variables from a many-dimensional FokkerPlanck equation. Physica A 130, 123 (1985).

12. Fesseha Kassahun. Refind Quantum Analysis of Light (Create Space Independent Publishing Platform, 2014).

13. T. Abebe. The quantum analysis of nondegenerate threelevel laser with spontaneous emission and noiseless vacuum reservoir. Ukr. J. Phys. 63, 969 (2018).

14. C.W. Gardiner, P. Zoller. Foundation of Quantum Optics (Imperial College Press, 2013).

15. F. Kassahun. Fundamentals of Quantum Optics (Lulu Press Inc., 2010).

16. M.O. Scully, M.S. Zubairy. Quantum Optics (Cambridge University Press, 1997).

Received 31.07.20

\section{М. Молла Гессессе}

\section{ВЗАЄМОДІЯ ДВОРІВНЕВИХ}

АТОМІВ 3 ОДНОМОДОВИМ КВАНТОВАНИМ

ПОЛЕМ ВИПРОМНЮЮАННЯ

Досліджуються статистичні властивості і стисливість світла в порожнині, випроміненого дворівневим лазером. Ця оптична система містить $N$ дворівневих атомів у порожнині, з'єднаній із одномодовим вакуумним резервуаром. Перехід атомів з нижнього на верхній рівень здійснюється під дією бомбардування електронами. Використовуючи стаціонарні розв'язки рівнянь еволюції для середніх значень операторів і квантове рівняння Ланжевена, ми знаходимо локальну і глобальну статистику одномодового світлового пучка. Показано, що для дворівневого лазера, який працює в режимі значно вище порога, невизначеності додатних i від'ємних квадратур рівні і задовольняють мінімуму співвідношення невизначеності. Тому світло від лазера, що працює вище порога, вважається когерентним. Але якщо лазер працює близько до порога, світло є хаотичним. 3 отриманих результатів ми також бачимо, що велика частина середньої локальної кількості фотонів, дисперсія їх числа і дисперсія локальної квадратури обмежені відносно вузьким інтервалом частот.

Ключов $i$ слова: середне число фотонів, дисперсія квадратури, вакуумний резервуар, шум. 